\title{
Mujeres, diferencias y multiplicidad. Apuntes para un abordaje ético de la vida subjetiva y corporal del género*
}

Nayla Luz Vacarezza**

\section{Resumen}

Este artículo propone una interrogación acerca de los modos en que es posible continuar utilizando, con propósitos académicos y políticos, la categoría identitaria "mujeres" desde una perspectiva que recoja los aportes del pensamiento crítico de la modernidad, con énfasis en la teoría de la performatividad del género propuesta por Judith Butler y en la filosofía de la multiplicidad de Gilles Deleuze. En lugar de apelar a un reparto trascendente y binario de los géneros optamos por una forma ética de mantener la categoría radicalmente abierta. Seguir la pista de la vida social y temporal presente de la categoría permite hacer visible un campo múltiple de subjetividades sociales que se ofrecen a otros desde una posición de enunciación y existencia corporal como "mujer".

Palabras clave: Mujeres, Multiplicidad, Diferencias, Performatividad del Género.

" Recebido para publicação em 16 de junho de 2010, aceito em 26 de maio de 2011.

** Docente e investigadora de la Facultad de Ciencias Sociales, Universidad de Buenos Aires, Argentina. Becaria doctoral del Consejo Nacional de Investigaciones Científicas y Técnicas (CONICET). nayla.luz@gmail.com

cadernos pagu (39), julho-dezembro de 2012:345-365. 
Mujeres, diferencias y multiplicidad

Woman, Differences and Multiplicity. Notes for an Ethical Approach to the Subjective and Bodily Life of Gender

\begin{abstract}
This article posits an interrogation about the ways in which it is possible to keep using, for academic and political purposes, the category "women" from a perspective that collects the contributions of the gender performativity theory proposed by Judith Butler, and the philosophy of multiplicity by Gilles Deleuze. Instead of appealing to a transcendent and binary gender distribution, we opt for an ethical way of keeping the term "women" as a radically open category. Following the track of the present social life of the category gives visibility to a multiple field of social subjectivities which offer themselves to others from a position of enunciation and corporal existence as "woman".
\end{abstract}

Key Words: Women, Multiplicity, Differences, Gender Performativity. 
"La variabilidad en la vida del término no excluye su utilización. Si un término se torna cuestionable, ¿̇significa eso que ya no se puede utilizar y que sólo podemos utilizar los términos que ya sabemos cómo dominar?" (J. Butler, Deshacer el Género)

"Cada categoría, cada universal, cada clasificación desconecta.

$\mathrm{O}$ es función de una desconexión. Para cada desconexión, una noción universal." (G. Deleuze, En medio de Spinoza)

Los universalismos junto con la noción de sujeto se cuentan entre los términos más asediados por el pensamiento crítico de la modernidad en cuyo desarrollo el feminismo ha tenido un rol protagónico incuestionable. La interrogación iniciática de Simone de Beauvoir acerca del estatuto social y político de la "mujer" se convirtió en uno de los debates animadores de la teoría y la política feminista hasta la actualidad. Aunque tempranamente quedó claro que la categoría "mujer" era insuficiente a la hora de referirse a un colectivo de "mujeres" fragmentado internamente por conceptos como clase, etnia, sexualidad, generación, por nombrar algunas, las disputas por lo que comprende (y con ello necesariamente excluye) el término "mujeres" en tanto categoría identitaria universalizante que presume alguna forma determinada de sujeto están lejos de ser saldadas. El agonismo en torno a la identidad fundamental que podría articular teoría y política, lejos de ser tranquilizadoramente suspendido ha sido, al interior del feminismo, ciertamente radical. ${ }^{1}$

La necesidad de someter a una exploración crítica las categorías centrales del feminismo ha sido muchas veces acusada

1 En un debate todavía abierto, cuyo examen excede los alcances de este trabajo, se han sostenido con fundados argumentos tanto a) la necesidad de abrir la categoría "mujeres" para que emerjan en su interior otras diferencias como pueden ser la raza, etnia, sexualidad, generación, etc. b) la inexistencia de las "mujeres" por fuera del régimen falocéntrico y la necesidad de refundar la cultura y el lenguaje en términos específicamente femeninos (L. Irigaray), c) la pertinencia de descartar definitivamente el término "mujer" o cualquier otro que aluda a la diferencia sexual (M. Wittig). 
de horadar las posibilidades de construir saber válido y de articular acciones políticas. Este artículo se posiciona en el interior de dicho problema y plantea una interrogación crítica que conserva provisionalmente la categoría identitaria en cuestión, principalmente por reconocer el poder subjetivante que posee en nuestro presente sociohistórico. Nos preguntamos por los modos en que la categoría "mujer" es recreada cotidianamente por quienes la utilizan para presentarse socialmente. Esbozaremos aquí esta perspectiva recogiendo aportes del pensamiento crítico de la modernidad, con énfasis en la teoría de la performatividad del género propuesta por Judith Butler y la filosofía de las diferencias y la multiplicidad de Gilles Deleuze.

Intentaremos avanzar sobre una serie de interrogantes relacionados con el problema planteado, ¿es posible someter a revisión crítica y al mismo tiempo utilizar los términos que se cuestionan? ¿Qué futuros pueden abrirse a partir de la utilización presente de nociones abiertas y contingentes de la categoría identitaria "mujeres"? ¿Qué posibilidades se movilizan al utilizar y optar por el término "mujeres" en un sentido ético, por contraposición a un sentido moral?

Siguiendo la lectura que G. Deleuze hace de B. Spinoza (Deleuze, 1975, 2004, 2008), si se quiere eludir los imperativos morales que acarrean las categorías universalizantes es necesario conocer los modos de existencia singulares. Convertir a la categoría "mujeres" en una opción ética significa, en la perspectiva que proponemos, suspender el sentido moral que enlaza de manera normativa a los sujetos con rasgos esencialistas. El régimen moralizante de verdad acerca de los géneros impone una cantidad de exigencias sobre los cuerpos y presupone que cada "mujer" posee - entre otros atributos- un tipo específico de genitalidad, unos caracteres sexuales secundarios (busto prominente, caderas anchas, escaso vello corporal), un deseo sexual (heterosexual) por el otro género y una práctica sexual orientada a la reproducción. 
Un tipo de aproximación apropiada a la vida contemporánea del género no puede partir de estas perimidas aunque resistentes exigencias universalistas. Por el contrario, entendemos que es necesario comenzar por la experiencia de quienes viven, utilizan y escapan de las categorías de la identidad de género. Ubicados en el plano ético que proponemos no sería válido ningún juicio guiado por criterios a priori para establecer la pertenencia a un género. En lugar de una totalización que capture las particularidades subsumiéndolas a los imperativos del "ser mujer", proponemos seguir la pista de las apropiaciones cotidianas de ese término identitario. Se podría así dar cuenta de la persistencia y poder subjetivante de la categoría identitaria "mujer" y al mismo tiempo visibilizar el surgimiento de géneros alternativos que se apropian en sus hablas del femenino gramatical y de las cualidades corporales culturalmente asociadas con la feminidad.

\section{Universalidades abiertas}

Las nociones abiertas y contingentes de la universalidad elaboradas en el marco de la teoría política pueden sumar elementos para el tipo de reflexión que se está planteando. Especialmente resultan útiles las elaboraciones de E. Laclau y Ch. Mouffe (Laclau, 1996; Mouffe, 1999; Laclau y Mouffe, 2006) respecto de la necesidad de reflexionar acerca de la distinción universalismo-particularismo en términos de relaciones asimétricas pero íntimas y no excluyentes. ${ }^{2}$

Según estos autores que rechazan las visiones esencialistas de la identidad, la afirmación y articulación de cualquier identidad particular supone una aspiración universalizante que pone en funcionamiento una "lógica equivalencial" que suspende las diferencias entre las particularidades internas al sistema.

\footnotetext{
${ }^{2}$ Un profundo y extenso debate en torno a estos temas puede encontrarse también en Contingencia, hegemonía y universalidad. Diálogos contemporáneos en la izquierda de Judith Butler, Ernesto Laclau y Slavoj Zizek (2003).
} 
Mujeres, diferencias y multiplicidad

Concomitantemente, cada articulación equivalencial funda una "lógica de la diferencia" que señala los límites del sistema, es decir, el exterior constitutivo más allá del cual no hay equivalencia posible. La idea de exterior constitutivo, que toman del pensamiento de J. Derrida, ocupa un lugar decisivo en sus argumentos pues indica que la condición de existencia de toda identidad es la afirmación de una diferencia constitutiva que es contingente pero imposible de erradicar.

Resultaría apropiado, en este marco, reflexionar sobre la categoría identitaria en cuestión como si se tratara de un significante vacío, es decir, un término sin significado ni contenido concreto a priori que señala la imposibilidad estructural inherente a todo intento de fijación identitaria. Si entendemos a la categoría identitaria como un particularismo diferencial que tiende a una universalización relativa, estamos aludiendo a un lugar vacío que señala el cruce entre una "lógica equivalencial" que cancela las diferencias internas a la categoría y una "lógica de la diferencia" respecto de aquello que forma su exterior constitutivo. Entonces, cuando invocamos dicha categoría tenemos que aceptar que estamos aludiendo a un compromiso inestable entre equivalencia y diferencia que es el resultado de una articulación hegemónica contingente e histórica, siempre disputada y abierta tanto a la rearticulación como a la desarticulación.

En nuestro presente histórico regional podría decirse que los compromisos en torno a la categoría identitaria "mujer" y al sistema de diferencias de género se encuentran en pleno proceso de renegociación. Un ejemplo de ello es la visibilidad y fortaleza creciente de las demandas del activismo trans ${ }^{3}$ por el reconocimiento y acceso a una ciudadanía plena de quienes no

3 Tanto en el ámbito del activismo como en el de los estudios sobre géneros y sexualidades suele utilizarse el término trans para hacer referencia a una multiplicidad de posiciones subjetivas que han elegido una identidad de género diferente de la atribuida al nacer. El término incluye a las subjetividades travestis, transexuales, transgéneros y otras autodenominaciones relacionadas (Sabsay, 2011:60). 
permanecen dentro de los rígidos límites que imponen las categorías del género.

La sanción de leyes que reconozcan la identidad de género autopercibida y que permitan tanto realizar modificaciones corporales como rectificar los datos personales en la documentación oficial evitando diagnósticos patologizadores es uno de los principales objetivos políticos de este colectivo. ${ }^{4} \mathrm{La}$ articulación pública de esta demanda significa un profundo desplazamiento en los modos en que el género es asignado. Tal es el caso de Argentina donde en 2012 se sancionó una Ley de Identidad de Género de avanzada a nivel mundial que responde a estos principios. Aun cuando dicha ley mantiene todavía al "sexo"/género como un sistema dicotómico, abre la posibilidad para que las categorías identitarias dejen de ser asignadas por criterios médicos refrendados jurídicamente para convertirse en una posible elección que refleje la experiencia personal, corporizada y social de cada quien.

Como adelantamos, no es el objetivo de este trabajo reponer una consistente y tranquilizadora categoría identitaria "mujer" sino exponerla a un examen crítico acorde a nuestro presente histórico en el cual las fronteras entre los géneros se hacen cada vez más porosas. No se intenta descartar o abolir las categorías de la identidad de género por considerarlas inseparables de la normativa heterosexista con el objetivo de fundar un nuevo orden de los géneros, con nuevas categorías o prescindiendo de ellas. Se trata, y en esto acordarían tanto J. Butler como E. Laclau y Ch. Mouffe, de utilizar políticamente los términos de la identidad con los que contamos pero posponiendo su contenido, dando cuenta de que se encuentran en un proceso

4 Estas demandas están sustentadas en los Principios de Yogyakarta que establecen cómo se aplica la legislación internacional de derechos humanos a las cuestiones de orientación sexual e identidad de género. Ver http://www.yogyakartaprinciples.org/principles_sp.htm. 
Mujeres, diferencias y multiplicidad

social de rearticulación constante y abiertos a un futuro imposible de anticipar:

Al admitir la provisionalidad estratégica del signo (en vez de su esencialismo estratégico) esa identidad se puede convertir en un espacio de cuestionamiento y revisión, adquirir un futuro conjunto de significados que tal vez ninguno de los que en este momento lo utilizamos seamos capaces de prever. Es en esta salvaguarda del futuro de los significantes políticos - preservando el significado como un espacio para la rearticulación - donde Laclau y Mouffe ven su potencial democrático (Butler, 2000:95).

En este marco, y siguiendo los planteamientos de avanzada iniciados por J. Butler en El género en disputa (Butler, 2001a) respecto de la necesidad de que el feminismo no abandone el trabajo autocrítico respecto de su propio sujeto, correspondería preguntarse, ¿qué cuerpos y subjetividades pueden ocupar legítimamente, en las condiciones actuales, un lugar entre las "mujeres" llenando precariamente este significante vacío? ¿Qué luchas agonistas se libran en el interior y en los márgenes de la lógica equivalencial que inaugura la categoría? ¿Qué exclusiones fundan la categoría identitaria "mujer" tal como la conocemos en la actualidad? ¿Qué cuerpos y subjetividades se apropian de ese término y al usarlo lo arrastran más allá de sí mismo?

\section{Performatividad del género}

Como dijimos, un modo de mantener abierta la categoría identitaria "mujer" podría consistir en seguir la pista de sus usos en el presente histórico de nuestra vida social. Podríamos preguntarnos, en este sentido, quiénes y cómo utilizan la categoría al vivir como "mujer", asumiendo en su habla un posicionamiento gramaticalmente femenino $y / 0$ corporizando los atributos culturalmente asociados con la feminidad. Esta operación permite hacer visible un campo amplio, plural y fluido de cuerpos $y$ 
subjetividades sociales que son difícilmente reducibles a las categorías discretas del género pero que las toman para sí y las utilizan para reconocerse a sí mismas y presentarse socialmente.

En el contexto de la teoría de la performatividad del género elaborada por J. Butler es justamente el uso ritualizado de los términos normativos del género lo que los produce y reproduce como realidad pero también lo que los abre a la temporalidad y a una vida material que, aun impregnada siempre y desde el principio por los poderes, los deja constantemente expuestos al cambio. La figura del travestismo (drag) es utilizada ya en sus primeras formulaciones (Butler, 1998, 2001a) para exponer la contingencia involucrada en los procesos de repetición necesarios para hacerse un lugar dentro del sistema del género. La experiencia generizada de las travestis -quienes se valen de ciertas características asociadas con lo femenino para presentarse socialmente- muestra de un modo radical que el proceso imitativo que instituye el género no se desprende de ninguna verdad originaria (como podría ser el "sexo") sino que se produce en la superficie del cuerpo y en la exterioridad de habla.

Ahora bien, la "actividad" de tomar para sí los atributos sociales del género en el contexto de la crítica del sujeto en la que se inscribe $\mathrm{J}$. Butler necesita de algunas necesarias aclaraciones porque su apuesta teórico-política es justamente eludir la dicotomía entre voluntarismo individual y determinismo social. Las prácticas de repetición paródica de las travestis muestran que las normas no determinan los géneros vividos, pero es necesario agregar que el género no es actuado por una voluntad soberana. Para J. Butler, que en este punto se reconoce deudora del pensamiento de L. Althusser y M. Foucault, no hay ningún sujeto que preceda a la normativa que lo habilita como tal porque ésta es previa al sujeto y al "yo" que existe sólo como efecto del poder y de la interpelación de las normas que lo inscriben en un mundo que lo excede y lo precede. Así, no podría pensarse en un sujeto capaz de expresar su voluntad respecto del género valiéndose de la normativa genérica como podría hacerlo de una herramienta. 
Mujeres, diferencias y multiplicidad

La citación de las normas del género es obligatoria para que una persona sea un sujeto socialmente viable pero no existe interpretación corporal que acate la norma o pueda encarnarla completamente. Las necesarias recapitulaciones locales que hacen a la existencia misma de la norma la exponen constantemente al riesgo de provocar efectos imprevistos, de transformarse o de ser interrumpida:

La práctica mediante la cual se produce la generización, la incorporación de normas, es una práctica obligatoria, una producción forzosa, aunque no por ello resulta completamente determinante. Puesto que el género es una asignación, se trata de una asignación que nunca se asume plenamente de acuerdo con la expectativa, las personas a las que se dirige nunca habitan por entero el ideal al que se pretende que se asemejen (Butler, 2002:324-325).

Los aportes de J. Butler permiten pensar en la inestabilidad constitutiva del sistema de género y en la posibilidad del cambio ya que toda acción social se despliega a través de la repetición y deja a las normas expuestas necesariamente a la temporalidad y a la diferencia. Los conceptos de citacionalidad $e$ iterabilidad retomados de las elaboraciones de J. Derrida son claves a la hora de comprender la forma en que J. Butler da cuenta del funcionamiento de las normas como sedimentación y acumulación de fuerzas realizadas a través de repeticiones que, aun siendo forzosas, al acontecer en un tiempo abierto e imposible de clausurar, se encuentran sujetas al desplazamiento:

Que los términos [femenino y masculino] sean recurrentes es bastante interesante, pero la recurrencia no indica una igualdad, sino más bien la manera por la cual la articulación social del término depende de su repetición, lo cual constituye una dimensión de la estructura performativa del género. Los términos para designar el género nunca se 
establecen de una vez por todas, sino que siempre están en proceso de estar siendo rehechos (Butler, 2004:25).

Aproximarnos desde esta perspectiva al estudio de las formas singulares en que las categorías del género son ambivalentemente impuestas y apropiadas en los rituales diarios de la vida corporal, permite advertir la miríada de intensidades y mezclas en los géneros que se producen en la práctica. Cada singularidad corporizada produce a través de repeticiones ritualizadas un género que la instituye, en los casos que nos interesan, como una "mujer" o una subjetividad "femenina". La identidad de género entonces no descansa en ninguna verdad originaria sino que se encuentra sostenida únicamente en repeticiones que deben ser sostenidas con el cuerpo y el habla para permanecer. El género debe ser analizado entonces como un tipo de práctica social e histórica que, aun cuando pueda adquirir una apariencia coherente y estable, siempre se encuentra expuesta a la posibilidad de desplazamientos e incongruencias, permitiendo todo tipo de variaciones de intensidad e incluso interrupciones a lo largo de una vida.

Reconocer el peso de la normativa de género como aparato anónimo de producción del binarismo mujer-varón y de una coherencia entre sexo, género y deseo no significa asumir que el género se reduzca a esa matriz heterosexual y binaria. Pensar a lo masculino y lo femenino como dominios claramente delimitables y excluyentes a través de los cuales se pueda dar cuenta de la vida social del género de manera exhaustiva es un gesto que consolidaría las expresiones normativas del género, reforzando una idealidad que debe ser cuestionada.

En este sentido, J. Butler sostiene que la idealidad binaria de las normas del género se impone mediante un efecto metaléptico ${ }^{5}$ producido por la sedimentación en el tiempo de las

5 La metalepsis es una figura retórica mediante la cual el consecuente es tomado por antecedente o viceversa. 
Mujeres, diferencias y multiplicidad

prácticas ritualizadas del género. Los que se toman como rasgos ideales del género son, por el contrario, producidos a través de actos ritualizados que son puramente externos $y$ derivados. Entonces, el género resulta desde esta perspectiva una realidad de superficies que produce lo interno y lo ideal como efecto.

$\mathrm{Si}$ atendemos a la autodesignación de las personas dentro del género femenino -sea a través del uso del género femenino gramatical en el discurso autorreferencial y/o en la presentación de atributos corporales culturalmente asociados con ese géneropodríamos dar cuenta de una multiplicidad de tácticas de supervivencia y estrategias corporales de "feminidades" o de "mujeres". Se hacen visibles entonces una variedad de subjetividades corporizadas que pueden haber sido o no asignadas a ese género al momento de su nacimiento, sin tampoco hacer distinciones a priori respecto de la pluralidad de formas que pueda adquirir su deseo sexual (es decir, sean heterosexuales, homosexuales, bisexuales o practiquen otras formas de sexualidad no catalogables identitariamente).

Un abordaje como el propuesto permite que la vida social de la categoría identitaria "mujer" se despliegue en su contingencia y que sus sentidos se dispersen en los actos particulares y corporizados que le dan vida. Podría entonces analizarse la producción social de lo "femenino" prestando atención a las prácticas generizadas de quienes han sido certificadas como "mujer" por los criterios médicos y jurídicos dominantes pero también incluirse en la reflexión a quienes experimentan formas de vivir la feminidad que han sido históricamente catalogadas como ilegítimas. En relación a quienes han sido asignadas como "mujer" al nacer, resulta necesario dar cuenta de los procesos rituales y cada vez más tecnificados mediante los cuales estos cuerpos se ven compelidos a reafirmar su "naturaleza femenina", por ejemplo, a través de distintos tipos de terapias cosméticas y quirúrgicas. Advertir las formas artificiosas en que se producen los géneros considerados legítimos aproxima estas experiencias a la de una cantidad de variantes de género que 
pueden manifestar distintos grados de incoherencia respecto de la norma: la feminidad de las travestis, de las transexuales y transgéneros, la feminidad de las transformistas, de las cross-dressers y de las drag queens, la feminidad de algunos varones y las expresiones de género de quienes se identifican como "mujer" pero prefieren renunciar a ciertos atributos asociados con la feminidad.

Si bien todos los géneros son construidos mediante actos de imitación, este proceso se encuentra políticamente regulado y fuertemente jerarquizado. Así es como algunas expresiones de género son consideradas normales, originales y verdaderas, mientras que otras son catalogadas como falsas, desviadas y/o patológicas. Denunciar y dar cuenta del modo en que la norma opera separando y jerarquizando las expresiones de género es un modo de extender el reconocimiento y de cambiar nuestra concepción de lo que es posible en la vida con género.

Sin embargo, la actitud crítica no supone establecer una nueva norma que clasifique cuáles son los géneros subversivos y cuáles los que refuerzan la normativa. J. Butler se ocupa de aclarar que la ruptura de la coherencia entre "sexo" y género que ponen en acto las subjetividades trans no es subversiva por sí misma, y que no debe ser tomada como un paradigma para la acción política. En el presente trabajo no proponemos la construcción de un juicio normativo sobre esta $\mathrm{u}$ otras variaciones del género sino que llamamos la atención sobre la "fluidez performativa" (Butler, 1998) propia de la práctica imitativa del género. Entender a los cuerpos con género como singularidades que se construyen a través de una imitación regulada permite hacer visible tanto la carga normativa que acarrea la categoría "mujer" y su relativa estabilidad como también los modos paradójicos en que ésta es aspirada, ocupada y recreada. 
Mujeres, diferencias y multiplicidad

\section{Multiplicidad y diferencia}

El feminismo puede comprenderse como una actividad teórica y política que de diversas maneras intentó pensar la diferencia y proponer herramientas siempre renovadas para reflexionar sobre ella. Como dijimos, no se trató sólo de la diferencia de las "mujeres" en tanto tales, es decir, de su especificidad respecto de los "varones", sino también de la diferencia creciendo y abriéndose paso al interior de ese colectivo. Consideramos que la filosofía de las diferencias y la multiplicidad de Gilles Deleuze, aun cuando no esté inscripta dentro del pensamiento feminista, puede aportar herramientas sugestivas para producir un abordaje pluralista y múltiple de lo que se postula como "mujeres". 6

En el trabajo filosófico de G. Deleuze puede observarse un obstinado esfuerzo por reflexionar acerca de la diferencia escapando de los binarismos opositivos y jerarquizados de la filosofía occidental. Su pensamiento puede entenderse como una afirmación de la diferencia entendida como multiplicidad proliferante e imposible de jerarquizar que se opone a la lógica de lo Mismo (también nombrada como lo mayoritario, lo molar o lo sedentario).

La división normativa hombre-mujer es, en los términos de G. Deleuze, una de las máquinas binarias que condicionan lo socialmente posible al nivel de la acción, la percepción, la afectividad y el pensamiento. ${ }^{7}$ Estas disyunciones suponen un

${ }^{6}$ Las elaboraciones de este apartado refieren especialmente al trabajo de G. Deleuze en colaboración con F. Guattari en Mil Mesetas (Deleuze y Guattari, 2008) y a las lecturas que G. Deleuze hizo de la filosofía de B. Spinoza (1975, 2004 , 2008). También recogemos aportes de la valiosa producción de otros autores que, dentro y fuera del feminismo, se reconocen como deudores de esta filosofía de la multiplicidad y de las diferencias (Braidotti, 2000, 2004, 2005; Gatens, 2000; Grosz, 1994; Lazaratto, 2006).

7 En el caso que nos interesa la disyunción varón-mujer, encerrada en la unidad heterosexista, permanece capturada dentro de la lógica de lo Mismo. Como esta, 
estado de poder y dominación a nivel social a través del cual se lleva a cabo un intento siempre renovado de encerrar la complejidad y la multiplicidad de lo social en realidades standard y estereotipadas. Lo mayoritario no indica una cantidad mayor sino un modelo normativo, es decir, un patrón ideal en relación al cual las singularidades se valoran.

Tanto el "hombre" como su otro complementario y especular, la "mujer", son lugares ontológicos ocupados por nadie, porque en la práctica social las singularidades siempre son variables y se distribuyen como minorías que son capturadas, pero también desbordan aquí y allá al modelo. Lo mayoritario funciona como un intento de universalización que, aun cuando es infructuoso por definición, no deja de limitar el campo de lo socialmente posible. Así, los sitios mayoritarios del género efectúan un trabajo de uniformización y universalización que tiene como objeto a lo minoritario, aquello que es objeto de capturas pero que no cesa de variar, y con ello huir de lo mayoritario que es un lugar vacío e inhabitable para cualquier singularidad.

El pensamiento de G. Deleuze resulta adecuado al tipo de enfoque que estamos proponiendo porque también intenta poner en variación lo que se presenta como constante y hacer proliferar las diferencias en el seno de la dicotomía entendida como oposición regulada y jerarquizada. En el modo de aproximarnos a las diferencias que estamos esbozando siguiendo a Deleuze, importan los umbrales, los arrastres y las conjugaciones que convierten, de hecho, al campo de los géneros en una multiplicidad minoritaria siempre reconducida por los aparatos binarios de codificación. En este sentido, es importante aclarar que lo minoritario no existe por fuera de su relación con lo mayoritario. La lógica de la proliferación y de la multiplicidad no necesariamente ignora el trabajo de lo negativo.

existen una cantidad de máquinas binarias: adulto-niño, humano-animal, blanconegro, intelectual-manual, heterosexual-homosexual, entre otras. 
Mujeres, diferencias y multiplicidad

Como en las elaboraciones de J. Butler, no se postula la posibilidad de devenires libres en un espacio liso sino la apertura de complejas trayectorias en un espacio estriado donde es necesario reconocer las potencias de sufrimiento y destrucción que se efectúan a través de los aparatos de captura del género binario. Pensemos, por ejemplo, en las distintas formas de violencia, exclusión y vulnerabilidad a la que son expuestas las personas trans y quienes habitan géneros incoherentes o confusos respecto de la norma.

Comprender a los géneros como una realidad que sólo puede ser parcialmente capturada por binarismo hombre-mujer permite cuestionar esa matriz de inmovilidad fundadora que se sustrae del juego de las diferencias. En el abordaje que proponemos se trata de conservar provisionalmente el término "mujer" para someterlo a un devenir minoritario que pueda dar cuenta de que su existencia se realiza a través de las múltiples variaciones singulares que lo encarnan y que constantemente se apropian, negocian y escapan a las categorías mayoritarias de la identidad y a los atributos que se le asocian.

En este sentido, lo común entre subjetividades que se presentan socialmente como "mujer" y/o se apropian de los rasgos culturalmente asociados con la "feminidad" no puede ser sujeto de una totalización sino de una composición conflictiva que sólo puede ser conocida enlazando singularidades. Descartar todo reparto de los géneros que pueda presentarse como originario o trascendente es el primer paso para construir un plano distributivo que se reacondicione en la experiencia y a la vista de la pragmática social e histórica de los géneros.

Si consideramos, siguiendo a G. Deleuze, que el conjunto no se define por sus constantes sino por las líneas de variación que lo componen, se podría reflexionar acerca de las "mujeres" y la "feminidad" como una zona de variación continua. Encontraremos entonces singularidades más o menos capturadas por los modos convencionales de la "feminidad" o de ser una "mujer". Algunas de ellas manifestarán una coherencia entre el 
"sexo anatómico", la identidad y la expresión de género, mientras que otras crearán combinaciones impensadas entre estos términos. Las primeras singularidades estarán empeñadas en un trabajo constante de territorialización y búsqueda de coherencia que les permita habitar un lugar ontológico imposible, mientras que otras actuarán como tensores y arrastrarán al sistema hegemónico de género hacia sus límites, desterritorializándolo. Puede advertirse la variabilidad que abre esta perspectiva, ya que no se reduce ni se reserva a aquellas manifestaciones que permanecen como disidentes, marginales o extraordinarias, sino que permite reflexionar acerca del trabajo ordinario $y$ cotidiano que compromete a todas las singularidades que se presentan socialmente como "mujer".

\section{Un plano ético para la indagación}

Volvemos en este punto al esfuerzo por ubicar a este trabajo en un plano ético donde los modos singulares de subjetivación como "mujer" puedan instalarse en un plano abierto donde sus diferencias se distribuyan como equivalencias. Para ello es necesario abandonar toda perspectiva que suponga un descenso mecánico desde la idealidad trascendente de las normas del género hacia la materialidad de los modos corporales de subjetivación propios de la vida con género. Diluir esta jerarquía permite reflexionar acerca de la productividad de lo normativo en un campo material sin exterior, esto es, no otorgar ningún privilegio al poder restrictivo de las normas del género sino atender a sus efectos productivos en términos de subjetividades y cuerpos. ${ }^{8}$

\footnotetext{
8 En sus últimos trabajos J. Butler desarrolla una concepción de la norma como una causa inmanente de lo social. Específicamente se ocupa este tema en el capítulo "El reglamento del género" perteneciente al libro Deshacer el género (Butler, 2004). Esta perspectiva, deudora de la filosofía inmanentista de Spinoza, también puede reconocerse en los trabajos de Michel Foucault y la filosofía de Gilles Deleuze, que incluye sus trabajos en colaboración con Félix Guattari.
} 
Podría decirse, siguiendo estas líneas de argumentación, que el género es una realidad accesible primordialmente en sus actualizaciones, en las múltiples prácticas locales que posibilita y constriñe. Por lo que resulta necesario detenerse a pensar cómo las normas actúan sobre las subjetividades corporizadas que, al mismo tiempo, recapitulan y reproducen las normas que actúan sobre ellas. Se trata entonces de entender a las normas y al poder definiéndose a través de su efecto productivo de subjetividades y cuerpos que son singulares y, a la vez, múltiples en sus diferencias.

Las singularidades con género que interesan a la reflexión que proponemos se ofrecen a otros desde una posición de enunciación y existencia corporal como "mujer". Pero tal categoría, desde la perspectiva propuesta, no proviene de un reparto natural o trascendente sino que se configura a partir de las singularidades individuadas que se valen de los atributos culturalmente asociados con la feminidad para presentarse socialmente ante otros. Este abordaje permite imaginar el campo de los cuerpos con género de "mujer" como una multiplicidad de singularidades que, siguiendo a J. Butler, performativamente se hacen, deshacen y rehacen a través de un complejo trabajo de improvisación en un escenario constrictivo minado por el poder.

Una existencia corporizada con género femenino es el sitio para una diferencia tal que lo que es peculiar de cada quien en relación a ese género no es algo voluntario o consciente sino una forma de ser condicionada por un juego social del que es imposible evadirse pero que es, a la vez, imprevisible. Estas variaciones pueden considerarse también en términos de intensidad, lo que permite dar cuenta de la existencia de feminidades vagas, confusas o de rasgos notoriamente masculinos pero también de feminidades hiperbólicas, excesivas y pertinaces que a través de la actuación hiperbólica de la norma revelen lo tenue de la realidad del género. Todas las variaciones, que deben incluir también el rol de la sexualidad en la dinámica del género y son imposibles de adelantar exhaustivamente, se reparten en un 
plano intensivo de variación continua con sus grados máximos y mínimos de desterritorialización.

Las singularidades estudiadas que tengan una posición incoherente, abiertamente ambigua o no convencional respecto de la distribución mayoritaria de los géneros coincidirán en transitar espacios sociales de patologización, discriminación y repudio. Esta realidad plantea, como lo señala $\mathrm{J}$. Butler, problemas éticos y políticos relacionados con las formas diferenciales en que se distribuye el reconocimiento a nivel social. ¿Quiénes podrán ser reconocidas como "mujer" y quiénes, debido a lo inadecuado de su feminidad, permanecerán en la opacidad del régimen de verdad de los géneros? ¿Qué nuevos géneros se están creando mediante la utilización de "viejas" categorías y estilos corporales?

Se trata de preguntas políticamente candentes a nivel global y especialmente en nuestra localización regional donde actualmente se fortalece el debate social acerca de la libre decisión de las personas sobre su propio cuerpo y sexualidad. Así también se multiplican las luchas que se dirigen a generar un cambio cultural amplio que extienda las posibilidades vitales de quienes, por diferentes motivos, han habitado hasta ahora los márgenes de las normas sexuales y de género. Nos referimos, entre otras, a la demanda de leyes que legalicen el aborto y garanticen el derecho a decidir sobre la propia vida sexual y reproductiva, que permitan el matrimonio entre personas del mismo sexo, que respeten la identidad de género autopercibida y que sostengan los cambios corporales de quienes los requieran, al mismo tiempo que impidan las cirugías compulsivas en personas intersex. Todas estas luchas socavan el sistema heterocentrado del género y la estabilidad de sus categorías porque cqué sucede con la categoría "mujer" cuando, mediante el ejercicio de la anticoncepción y del aborto, deja de ser equivalente a "madre"? ¿Qué desplazamientos afectan a las categorías del género cuando una "mujer" decide llevar adelante su vida reproductiva en comaternidad con otra "mujer"? ¿Puede una "mujer" tener pene o desear extirparse el busto? 
Mujeres, diferencias y multiplicidad

La multiplicidad de modos en que el género es experimentado - tanto dentro como fuera de la norma demuestra que habitamos un tiempo histórico en el cual el término identitario "mujer" no puede ya ser sostenido por criterios a priori. Por eso, planteamos esta reflexión como el ejercicio de un modo de conocimiento comprometido con las diferencias y también con la articulación de coaliciones políticas por venir. Un pensamiento y una práctica política que sean indisociables de una ética que pueda afirmar la heterogeneidad en este mundo, el de las categorías que tenemos y con las que ordinariamente se realiza lo extraordinario.

\section{Referencias bibliográficas}

BRAIDOTTI, Rosi. Metamorfosis. Hacia una teoría materialista del devenir. Madrid, Akal, 2005.

- Feminismo, diferencia sexual y subjetividad nómade. Barcelona, Gedisa, 2004.

. Sujetos nómades. Buenos Aires, Paidós, 2000.

BUTLER, Judith. Actos performativos y constitución del género: un ensayo sobre fenomenología y teoría feminista. Debate feminista (18). México, 1998, pp.296-314.

. Imitación e insubordinación de género. Revista de Occidente (235), Madrid, Fundación Ortega y Gasset, 2000, pp.85-109.

El género en disputa. El feminismo y la subversión de la identidad. Buenos Aires, Paidós, 2001a.

. Mecanismos psíquicos del poder. Teorías sobre la sujeción. Madrid, Cátedra, 2001b.

. Fundamentos contingentes: el feminismo y la cuestión del "posmodernismo". La Ventana (13), Guadalajara, Centro Universitario de Ciencias Sociales y Humanidades de la Universidad de Guadalajara, 2001c, pp.7-41. 
- Cuerpos que importan. Sobre los límites materiales y discursivos del "sexo". Buenos Aires, Paidós, 2002. . Deshacer el género. Barcelona, Paidós, 2006.

BUTLER, Judith; LACLAU, Ernesto; ZIZEK, Slavoj. Contingencia, hegemonía y universalidad. Diálogos contemporáneos en la izquierda. Buenos Aires, FCE, 2003.

ChAneton, July. Género, poder y discursos sociales. Buenos Aires, EUdeBA, 2007.

Deleuze, Gilles. En medio de Spinoza. Buenos Aires, Cactus, 2008. . Spinoza: Filosofía práctica. Buenos Aires, Tusquets, 2004. Editores, 1975. . Spinoza y el problema de la expresión. Barcelona, Muchnik

Deleuze, Gilles; GuatTARI, Félix. Mil Mesetas. Valencia, Pre-Textos, 2008.

DERRIDA, Jacques. Márgenes de la filosofía. Barcelona, Anthropos,1989.

GATENS, Moira. Feminism as "password": Re-Thinking the "possible" with Deleuze and Spinoza. Hypatia (15-2), Indianápolis, Indiana University Press, 2000, pp.59-75.

GROSZ, Elizabeth. Volatile bodies. Towards a corporeal feminism. Bloomington, Indiana University Press, 1994.

LACLAU, Ernesto. Emancipación y diferencia. Buenos Aires, Ariel, 1996.

LACLAU, Ernesto; MoufFe, Chantal. Hegemonía y estrategia socialista. Buenos Aires, FCE, 2006.

LazAratto, Maurizio. Políticas del acontecimiento. Buenos Aires, Tinta Limón, 2006.

MoufFe, Chantal. El retorno de lo político. Comunidad, ciudadanía, pluralismo, democracia radical. Barcelona, Paidós, 1999.

NichOlsON, Linda. Feminismo/Posmodernismo. Buenos Aires, Feminaria, 1992.

SABSAY, Leticia. Fronteras sexuales. Espacio urbano, cuerpos y ciudadanía. Buenos Aires, Paidós, 2011. 